\title{
Darier's disease: hopes and challenges
}

\author{
Laurence Hulatt BA Susan Burge DM FRCP ${ }^{1}$
}

J R Soc Med 2003;96:439-441

In an article in the JRSM 14 years ago, SB described the predicament of a patient with Darier's disease (keratosis follicularis): 'His scalp is crusted, the skin around his ears weeps and scales ... the odour which shadows him pervades the immediate environment and remains when he has gone'. She concluded, 'Perhaps by the end of this century it will be possible to describe precisely what has happened in the skin in Darier's disease and this will result in improved therapy so that our patients can lead a normal life.' ${ }^{1}$ We now discuss the progress made in the intervening years, as it bears on the case of another patient, AB.

$A B$ 's disease is extensive, covering the trunk, face, neck and scalp; and severe, being refractory to conventional medical therapy. Erosive flexural disease in the natal cleft causes painful fissuring and secondary infection. Surgery has been attempted with limited success; he still suffers pain on sitting down. Of particular interest are the views of $\mathrm{BB}$, his mother. She herself has mild disease, but seems to view her son's disease as being more severe than he himself does. Furthermore, she feels great responsibility for her son's condition, expressing feelings of guilt: 'I would never have had children if I had known it could do this....

\section{CLINICAL FEATURES}

Darier's disease is a dominantly inherited condition affecting skin, nails and mucosae. ${ }^{2}$ Prevalence is estimated at 1 in 36000 (in North-East England). ${ }^{3}$ Skin signs generally develop between 6 and 20 years of age, although lesions may be overlooked until aggravated by heat, sweating or sunlight.

Clinically, the distinctive lesion is a warty plaque formed by coalescing firm, greasy, skin-coloured papules (Figure 1). The seborrhoeic areas of the trunk and face are predominantly affected. Pruritus is common, occurring in $80 \%$ of patients, and may be intractable; pain is unusual. When flexures are involved, the lesions may be hypertrophic, fissured and malodorous.

Oxford University Medical School and ${ }^{1}$ Department of Dermatology, Churchill Hospital, Oxford, UK

Correspondence to: Dr S M Burge, Department of Dermatology, Churchill Hospital, Headington, Oxford OX3 7LJ, UK

E-mail: sue.burge@ndm.ox.ac.uk
Darier's disease runs a chronic course and may worsen with age. Secondary infection of skin lesions is a common complication. Most patients do not have any co-morbid ills. $^{2}$

\section{PATHOGENESIS}

Histologically, loss of cohesion between suprabasal epidermal cells (acantholysis) results in suprabasal clefting with papillomatosis and dyskeratosis. Electronmicroscopy shows loss of the desmosomal protein attachments that normally link keratinocytes and perinuclear aggregation of keratin filaments, and immunohistological techniques indicate that in acantholytic cells desmosomal proteins are distributed diffusely within the cytoplasm. ${ }^{4,5}$ It has been suggested that the primary abnormality lies within the desmosomal plaque. ${ }^{6,7}$

The mutations responsible for Darier's disease have been located in the ATP2A2 gene on chromosome 12q2324.1. ${ }^{8}$ Mosaicism for ATP2A2 mutations causes segmental Darier's disease. ${ }^{9}$ This gene encodes a sarco/endoplasmic reticulum $\mathrm{Ca}^{2+}$ ATPase (SERCA2), which transports $\mathrm{Ca}^{2+}$ from the cytosol back to the endoplasmic reticulum lumen. ${ }^{10}$ Abnormalities of SERCA2 in Darier's disease may alter cell signalling and affect the synthesis, folding or trafficking of desmosomal components.

Various mutations are seen in Darier's disease and it has been proposed that the common mechanism is via haploinsufficiency: production of the normal phenotype requires

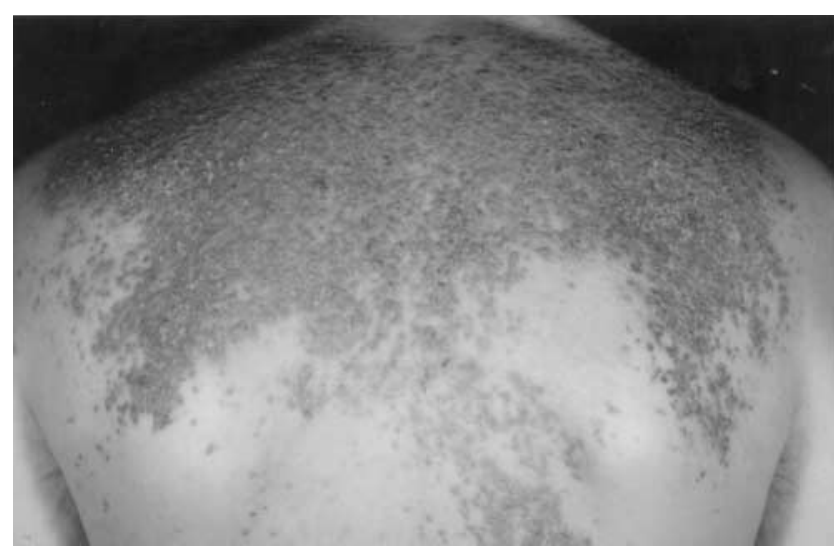

Figure 1 The itchy malodorous hyperkeratotic papules of Darier's disease, coalescing into plaques. 
more gene product than can be produced by a single copy of the gene. ${ }^{8}$ Histologically the lesions of Darier's disease show proliferative epidermal 'budding' and dyskeratosis as well as loss of adhesion. Desmosomes may participate in cell signalling and influence cell growth and differentiation. ${ }^{11}$ Knockout and mis-expression of desmosomal adhesion proteins (cadherins) in mice affect epidermal differentiation ${ }^{12,13}$ and, of relevance to Darier's disease, aged heterozygote SERCA2( \pm ) mice develop squamous epithelial papillomas and carcinomas. ${ }^{14}$

Unfortunately it is not possible to predict the severity of disease in the offspring of an affected person, and the outcomes of investigations into genotype-phenotype correlations have been disappointing. ${ }^{8}$ Considerable variation in severity was found within and between families, and in some patients with classic disease no mutation was identified. Clinical heterogeneity (a range of phenotypes produced by the same mutation) and genetic heterogeneity (identical phenotypes produced by different mutations) are well described in the genodermatoses. ${ }^{15}$ Explanations might include influences of modifying genes or the environment on phenotype. Detailed clinical studies of large numbers of individuals with Darier's disease may identify previously unrecognized subtle variations in phenotype associated with specific mutations. The imprecise nature of genotypephenotype correlation in Darier's disease is important when we come to consider the impact of mutation analysis on genetic counselling.

\section{TREATMENT}

Despite much progress in understanding of the underlying abnormality in Darier's disease, disappointingly little has changed in our treatment armoury. Conventional therapy for severe disease still relies greatly on oral retinoids. The clinical response is good in 90\% of patients: hyperkeratosis is reduced and papules are flattened, while malodour may also improve. However, retinoids are teratogenic and pregnancy must be avoided during and for a while after treatment. Common dose-related side-effects are mucosal dryness, nosebleeds, itching and photosensitivity, and these may be so troublesome that patients prefer to live with their disease. ${ }^{16}$ Topical retinoids may reduce hyperkeratosis in three months, but irritation is a common side-effect. ${ }^{16}$

Further unravelling of the molecular basis of Darier's disease may explain precisely why drugs such as retinoids are effective, and also why disease can be triggered by lithium, sweating or ultraviolet light. Progress will probably depend on studies that specify the molecular mechanisms linking the genetic defects with the observed phenotype. Repair of the disease gene in Darier's disease is a distant hope.

\section{GENETICS AND ETHICS}

\section{Genetic counselling}

Gene identification has considerable implications in Darier's disease. Should patients be offered genetic counselling, prenatal diagnosis and the possibility of terminating pregnancies that would result in the birth of a child with Darier's disease? Genetic counselling has been described as 'a communication process which deals with the human problems associated with the occurrence (or risk of) a genetic disorder in a family'. ${ }^{17}$ Genetic services stress the non-directiveness of the process, aiming to provide the best possible circumstances to allow an informed choice. Issues surrounding confidentiality and disclosure of information have come to the fore within genetic counselling services, since information obtained about one person has direct relevance to other members of the family; however, since Darier's disease is a dominantly inherited condition and non-penetrance is unusual, difficulties of this sort are rare and will not be discussed here.

The primary objectives of prenatal diagnosis as set out by the Royal College of Physicians stress the importance of providing informed choice, reassurance and the option of selective termination if a child is likely to be severely affected. ${ }^{17}$

Prenatal diagnosis has been used in the identification of skin disease since the $1980 \mathrm{~s} .{ }^{18}$ The original techniques involved morphological and biochemical examination of established fetal skin obtained by ultrasound guided skin biopsy in the second trimester. DNA-based prenatal diagnosis now allows diagnosis from fetal cells obtained via chorionic villus sampling (10 weeks) or amniocentesis (13 weeks). Also in-vitro fertilization techniques offer the possibility of pre-implantation genetic diagnosis of embryos at the 4-10 cell stage, avoiding the need for termination. Genetic defects may be identified and only disease-free embryos are selected for transfer to the uterus. ${ }^{18}$

\section{Ethical considerations}

Ethical principles are applied to genetic technology in much the same way as in other areas of medicine - for example, the balance of benefit versus harm. However, genetic choices are far more likely to touch the lives of others; indeed, interventions may lead to the replacement of one life with another. For those who accept that there are some circumstances where termination is morally justified, the argument will turn partly on the seriousness of the condition; there must be a minimum level of severity below which the case for termination becomes untenable.

Parents naturally want a 'perfect child', and some workers in genetic medicine fear a slippery slope of increasing intervention to remove mild or trivial disease or 
to select favoured characteristics. In truth, however, most of the public are opposed to human genetic engineering for any purpose other than to cure disease. ${ }^{19}$

Assessment of severity may be of particular difficulty in skin disease, especially when psychosocial aspects are encompassed in the decision. Clinicians base their assessments on history and examination. Relevant questions in the history include those relating to the symptoms of the disease and its impact on employment and social life. Examination focuses on the character of the lesions and their distribution. How closely these methods reflect what patients themselves think about their disease is open to doubt. Harris et al. ${ }^{20}$ found that physicians' assessment of Darier's disease severity correlated poorly with scores on a patient questionnaire, the Dermatology Life Quality Index. ${ }^{20}$ Some patients were severely handicapped by mild disease, others faring well with severe disease. Overall scores averaged 5.89, corresponding to $20 \%$ of the maximum. This may be compared with $30 \%$ in psoriasis and $24 \%$ in dystrophic epidermolysis bullosa, a severe blistering disorder in which DNA-based prenatal diagnosis is available. ${ }^{21-23}$

It has been suggested that standards should be set centrally, stating what constitutes sufficient severity for prenatal diagnosis to be offered. Sometimes, perhaps, the severity is so low that direct resources should be reserved for other more serious conditions. However, this approach raises further difficulties even if we decide that the State has the right to impose such restrictions. In Darier's disease the genotype-phenotype correlation is weak, making it hard to predict how severely a child will be affected even if the mutation is detected. Also, the severity of a condition depends on the social context of the individual; thus, a caseby-case approach is necessary.

\section{CONCLUSION}

Many difficult questions need answering if we are to develop a consistent approach to the use of emergent genetic technology. Returning to the case of $\mathrm{AB}$, how would we feel about genetic counselling if this patient requested it before starting a family? Perhaps more pertinently, would we have offered prenatal diagnosis to his mother had it been technically feasible 30 years ago?

\section{REFERENCES}

1 Burge SM. Darier's disease, keratins and proteases: a review. J R Soc Med 1989;82:673-6

2 Burge SM, Wilkinson JD. Darier-White disease: A review of the clinical features in 163 patients. J Am Acad Dermatol 1992;27:40-50
3 Munro CS. The phenotype of Darier's disease: Penetrance and expressivity in adults and children. Br J Dermatol 1992;127:126-30

4 Burge S. Darier's disease- the clinical features and pathogenesis. Clin Exp Dermatol 1994;19:193-205

5 Hakuno M, Shimizu H, Akiyama M, et al. Dissociation of intra- and extracellular domains of desmosomal cadherins and E-cadherin in Hailey-Hailey disease and Darier's disease. $\mathrm{Br} J$ Dermatol 2000; 142:702-11

6 Harada M, Hashimoto K, Fujiwara K. Immunohistochemical distribution of CD44 and desmoplakin I \& II in Hailey-Hailey's disease and Darier's disease. J Dermatol 1994;21:389-93

7 Hashimoto K, Fujiwara K, Tada J, et al. Desmosomal dissolution in Grover's disease, Hailey-Hailey's disease and Darier's disease. J Cutan Pathol 1995;22:488-501

8 Sakuntabhai A, Burge S, Monk S, et al. Spectrum of novel ATP2A2 mutations causes segmental Darier's disease. Hum Mol Genet 1999;8:1611-19

9 Sakuntabbai A, Dhitavat J, Burge S, et al. Mosaicism for ATP2A2 mutations causes segmental Darier's disease. J Invest Dermatol 2000; 115: 1144-7

10 MacLennan DH, Brandl CJ, Korczak B, et al. Amino-acid sequence of a $\mathrm{Ca} 2++\mathrm{Mg} 2+$-dependent ATPase from rabbit muscle sarcoplasmic reticulum, deduced from its complementary DNA sequence. Nature 1985;316:696-700

11 Green KJ, Jones JC. Desmosomes and hemidesmosomes: structure and function of molecular components. Faseb J 1996;10:871-81

12 Garrod D, Merritt A, Nie Z. Desmosomal cadherins. Curr Opin Cell Biol 2002;14:537

13 Merritt AJ, Berika MY, Zhai W, et al. Suprabasal desmoglein 3 expression in the epidermis of transgenic mice results in hyperproliferation and abnormal differentiation. Mol Cell Biol 2002;22:5846-58

14 Liu LH, Boivin GP, Prasad V, et al. Squamous cell tumors in mice heterozygous for a null allele of Atp2a2, encoding the sarco(endo)plasmic reticulum Ca2+-ATPase isoform $2 \mathrm{Ca} 2+$ pump. J Biol Chem 2001;276:26737-40

15 Irvine AD, McLean WH. The molecular genetics of the genodermatoses: progress to date and future directions. $\mathrm{Br} J$ Dermatol 2003;148:1-13

16 Cooper SM, Burge SM. Darier's disease: epidemiology, pathophysiology, and management. Am J Clin Dermatol 2003;4: 97-105

17 Steering group of the Medical Ethics Committee of the British Medical Association. Human Genetics: Choice and Responsibility. Oxford: Oxford University Press, 1998

18 Shimizu H, Suzumori K. Prenatal diagnosis as a test for genodermatoses: its past, present and future. $J$ Dermatol $S_{c i}$ 1999; 19:1-8

19 Elmer-Dewitt P. The genetic revolution. New technology enables us to improve on nature. How far should we go? Time, 17 January 1994

20 Harris A, Burge SM, Dykes PJ, Finlay AY. Handicap in Darier's disease and Hailey-Hailey disease. Br J Dermatol 1996;135: 959-63

21 Finlay AY, Khan GK. Dermatology Life Quality Index (DLQI) - a simple practical measure for routine clinical use. Clin Exp Dermatol 1994;19:210-16

22 Klingberg S, Mortimore S, Parkes J, et al. Prenatal diagnosis of dominant dystrophic epidermolysis bullosa, by COL7A1 molecular analysis. Prenat Diagn 2000;20:618-22

23 Horn HM, Tidman MJ. Quality of life in epidermolysis bullosa. Clin Exp Dermatol 2002;27:707-10 